\title{
Physiological and agronomic responses of maize (Zea mays L.) cultivars to plant population and defoliation at post-anthesis in the humid rainforest
}

\author{
Olalekan SAKARIYAWO ${ }^{1 *}$, Doyin OGUNDIRAN ${ }^{1}$, Paul SOREMI $^{1}$, Sunday ADERIBIGBE ${ }^{1}$
}

Received November 24, 2016; accepted July 12, 2018.

Delo je prispelo 24. novembra 2016, sprejeto 12. julija 2018.

\begin{abstract}
Variations in response pattern of maize (Zea mays) grown at plant populations, defoliated at post-anthesis in the rainforest were tested. Two field trials were conducted at Abeokuta, (Longitude $3^{0} 25^{\prime} \mathrm{E}$, Latitude $7^{0} 15^{\prime} \mathrm{N} ; 144 \mathrm{~m}$ a.s.l) and Ibadan $\left(3^{0} 56^{\prime} \mathrm{E}, 7^{0} 33^{\prime} \mathrm{N}\right.$ : $168 \mathrm{~m}$ a.s.l), Nigeria in 2015 . The trials consisted of maize variety 2009 TZE-W DT STR [open pollinated variety (OPV)] and TZEI $124 \times$ TZEI 25 (hybrid)] in the main plot, plant population $(71111,80000$ and 106666 plant $\mathrm{ha}^{-1}$ ) in sub plot and defoliation (+ defoliation and defoliation) as sub-sub plot. It was laid out in a split-split plot arrangement fitted into randomised complete block design with three replicates. OPV had significantly higher assimilatory surface, rate of current photosynthesis, reduced dry matter translocation efficiency, reduced days to $50 \%$ anthesis and more 1000 grain massthan the hybrid maize, with similar grain yields. Both locations experienced increased leaf area index with increased plant population. Reduced 1000 grain massat both locations when maize was defoliated suggested a disruption in source:sink balance.
\end{abstract}

Key words: defoliation; open pollinated maize; plant population; current rate of photosynthesis; efficiency of photosynthesis
IZVLEČEK

\author{
FIZIOLOŠKI IN AGRONOMSKI ODZIV SORT \\ KORUZE (Zea mays L.) NA GOSTOTO POSEVKA IN \\ DEFOLIACIJO PO ANTEZI V RAZMERAH \\ VLAŽNEGA DEŽEVNEGA GOZDA
}

$\mathrm{V}$ raziskavi so bile preiskuševane spremembe $\mathrm{v}$ odzivih koruze (Zea mays L.) na gostoto posevka in defoliacijo po antezi v razmerah vlažnega deževnega gozda. Izvedena sta bila dva poljska poskusa v Abeokuti (ZD $3^{0} 25^{\prime} \mathrm{E}, \mathrm{ZS} \mathrm{S}^{0} 15^{\prime} \mathrm{N}$; $144 \mathrm{~m}$ a.s.l) in Ibadanu ( $3^{0} 56^{\prime} \mathrm{E}, 7^{0} 33^{\prime} \mathrm{N}$ : $168 \mathrm{~m}$ a.s.l) v Nigeriji, leta 2015. Poskusi so obsegali sorto koruze \{2009 TZE-W DT STR [tujeprašno sorto (OPV)] in hibrid TZEI 124 $\times$ TZEI 25$\}$ na glavni ploskvi, gostoto posevka $(71111,80000$ in 106666 rastlin $\mathrm{ha}^{-1}$ ) na podploskvah in defoliacijo (+ defoliacijain - defoliacija) na nadaljnih podploskvah. Poskus je bil zasnovan kot popolni naključni bločni poskus $\mathrm{z}$ deljenkami s tremi ponovitvami.Tujeprašna sorta (OPV) je imela značilno večjo asimilacijsko površino, večjo fotosintezo, zmanjšano sposobnost translokacije suhe snovi, zmanjšano število dni do $50 \%$ anteze in večjo maso 1000 zrn kot hibridna sorta koruze, a podoben pridelek zrnja. Na obeh lokacijah se je indeks listne površine povečal $\mathrm{z}$ gostoto posevka. Zmanjšana masa $1000 \mathrm{zrn}$ na obeh lokacijah v primeru defoliacije nakazuje motnje $\mathrm{v}$ ravnovesju med virom in ponorom asimilatov.

Ključne besede: defoliacija; tujeprašna koruza; gostota posevka; velikost fotosinteze; učinkovitost fotosinteze

\section{INTRODUCTION}

Maize (Zea mays L.) is the most widely cultivated crop and the most important staple food in sub-Saharan Africa, accounting for up to $70 \%$ of the daily human calorie intake (Martin et al., 2000). One of the management factors that have contributed to the increased performance of maize in recent past was increased plant population. An appropriate plant stand may help in harnessing all the renewable and nonrenewable resources in a more efficient manner towards higher crop yields (Sarlangue et al., 2007). Unfortunately, there is no single recommendation for all conditions, because the optimum plant population varies depending on environmental factors such as soil fertility, moisture supply and genotype (Gonzalo et al.,

1 Department of Plant Physiology and Crop Production, College of Plant Science and Crop Production,Federal University of Agriculture, Abeokuta, P.M.B. 2240, Alabata, Ogun State, Nigeria, *corresponding author: adetanwa@ yahoo.co.uk 
2006). This management factor was able to increase productivity per unit area, albeit with reduced yield per plant. This observation could be attributed to the fact that increased plant population per unit area could predispose maize crop to increased competition for available growth resources, especially light and water (Lemcoff and Loomis, 1994).

It was also observed that with high plant population there could be a change in canopy architecture, attenuation of radiant energy incident on leavesand alternation in light spectrum(Maddonni et al., 2001). This could be accompanied by a reduction in red/far red ratio, increased leaf senescence and a reduction in canopy apparent photosynthesis (Sangoi, 2001). Suggestions had been made that defoliation of maize plant could reduce competition for light under increased plant density per unit area (Liu et al., 2015). This suggested technique would increase interception of light into the canopy thus increase yield per plant under high density (Liu et al., 2015). Defoliation of the two uppermost leaves in maize plant three days after silking had been reported in China to have resulted in significant improved performance of maize at high plant population (Liu et al., 2015). The efficacy of this technique is dependent on its timing, intensity, genotype used and environment (Ahmadi and Joudi, 2007; Yin et al., 1998; Zhenlin et al., 1998) and the intensity of defoliation. Defoliation could alter source-sink balance as manifested in a reduction in carbon assimilation process and subsequently reduced assimilate availability (Rajcan and Tollenaar, 1999). Assimilate availability could be mediated through remobilization of earlier formed assimilate to the sink especially during the grain filling period. This could result in increased kernel mass and increased maize productivity at high plant population. However, the possibility of increasing leaf senescence is high especially at the grain filling stage if maize crop experiences nitrogen deficiency (Borrás et al., 2003). Furthermore rate, efficiency and contribution of remobilization to assimilate availability under a compromised source-sink relationship could be further complicated by the type of maize involved. Generally it had been indicated that maize has low remobilization efficiency compared to soybean and wheat (Kiniry et al., 1992). There is paucity of information in the literature on the response of hybrid and open pollinated (OPV) maize to defoliation at grain filling stage and its implication on assimilate availability. Apart from the effect of defoliation on assimilate availability at the grain filling stage, it was reported that this could be further confounded by the amount of radiant energy available at this growth stage (Borrás et al., 2004). This is more germane in the transitory rainforest, where there had been a remarkable variation in weather pattern compared to what was obtainable in recent past. The implication of this environmental factor on maize (hybrid and OPV) types is still unknown. Maize hybrids are known to require high inputs to attain their yield potential. How this requirement would act in combination with the contribution of remobilization on the grain yield of hybrid maize compared with OPV on senescence at high plant density still needs further investigation.

This investigation tested the hypothesis that postanthesis defoliation of the two uppermost leaves could ameliorate the negative impact of increased plant population among maize cultivars; that there would be variation in the performance of these maize cultivars under these treatments in the rainforest transitory agroecology. Our findings would increase our understanding of the physiological mechanisms that underpins maize cultivar responses to defoliation at increasing plant population and would provide a technique of increasing plant population with minimal negative implication on their performance.

\section{MATERIALS AND METHODS}

\subsection{Description of location and experimental site}

The experimental fields were sited at the Teaching and Research farm, Federal University of Agriculture, Abeokuta (FUNAAB), Ogun state, (Longitude $3^{0} 25^{\prime} \mathrm{E}$, Latitude $7^{0} 15^{\prime} \mathrm{N}$; ; altitude $144 \mathrm{~m}$ a.s.l). and National Horticultural Research Institute, Ibadan (NIHORT) ( $3^{0} 56^{\prime} \mathrm{E}, \quad 7^{0} 33^{\prime} \mathrm{N}$ : altitude $168 \mathrm{~m}$ a.s.l), Nigeria. Agrometeorological data were collected from the agrometeorological station of FUNAAB and NIHORT. Soil particle size distribution was determined using the hydrometer method (Bouyoucos, 1962). Soil pH was determined in soil: water suspension (1:1) using glass electrode $\mathrm{pH}$-meter(McLean, 1982). Soil organic carbon was determined using the wet oxidation method of Walkley and Black (Allison, 1965). Total nitrogenwas determined using the modified micro Kjeldahl digestion technique (Jackson, 1962). Available phosphorus was evaluated based on Bray-1 method (Bray and Kurtz, 1945) and determined colometrically according to protocol devised Murphy and Riley, (1962). Exchangeable cations were determined by extracting the cation with $1 \mathrm{~N}$ ammonium acetatebuffered at $\mathrm{pH} 7$. Potassium in the extract was determined by flame photometry.At Abeokuta soil organic carbon was 0.53 mg kg-1, with $0.8 \mathrm{~g} \mathrm{~kg}^{-1}$ total nitrogen, $6.42 \mathrm{mg} \mathrm{kg}^{-1}$ available phosphorus and $\mathrm{K}^{+}$was $0.35 \mathrm{cmol} \mathrm{kg} \mathrm{kg}^{-1}$. At Ibadan the soil consisted of $0.46 \mathrm{mg} \mathrm{kg}^{-1}$ of organic 
carbon, $0.6 \mathrm{~g} \mathrm{~kg}^{-1}$ total nitrogen, $5.65 \mathrm{mg} \mathrm{kg}^{-1}$ of available phosphorus and $0.18 \mathrm{cmol} \mathrm{kg}^{-1}$ of $\mathrm{K}^{+}$. The soil textural class of both locations was sandy, while the preplanting soil $\mathrm{pH}$ at both locations was similar, slightly acidic (5.20 and 5.30 at Ibadan and Abeokuta respectively).

\subsection{Treatments and design}

The treatment consisted of maize cultivars, plant population and post-anthesis defoliation. These were arranged in split-split plot fitted into randomised complete block design. The main plot consisted of maize cultivars [TZEI $124 \times$ TZEI 25 (hybrid) and 2009 TZE-W DT STR (open pollinated)]. Both maize cultivars belong to the early maturity class (90-95 days to reach physiological maturity). The sub-plot was made of plant population $\left(106,666\right.$ plants $\mathrm{ha}^{-1}, 80,000$ plants $\mathrm{ha}^{-1}$ and 71, 111 plants $\mathrm{ha}^{-1}$ ), while the sub-sub plot consisted of post-anthesis defoliation (control and defoliated maize plant).

\subsection{Cultural practices}

Planting material was sourced from International Institute of Tropical Agriculture, Ibadan. Ploughing was done twice and harrowing was conducted once. Sowing of maize seed was done on a flat land surface.Planting was conducted manually at a depth of about $20 \mathrm{~mm}$ on $24^{\text {th }}$ and $25^{\text {th }}$ of May, 2015 at Abeokuta, $3^{\text {rd }}$ and $4^{\text {th }}$ of June, 2015 at Ibadan. Planting was conducted at a spacing of $0.75 \times 0.25 \mathrm{~m}$ at two plants per stand constituting 106,666 plants ha ${ }^{-1}, 0.75 \times 0.50 \mathrm{~m}$ at three plants per stand constituting 80,000 plants ha ${ }^{-1}$ and 0.75 $\times 0.75 \mathrm{~m}$ at four plants per stand constituting 71,111 plants $\mathrm{ha}^{-1}$. Each gross plot measured $3 \times 4 \mathrm{~m}$ with a net plot of $2 \times 2 \mathrm{~m}$. Plots were separated from each other by $0.5 \mathrm{~m}$ path and each block was separated by $1 \mathrm{~m}$ walk way.

Missing stands were supplied at 1 week after planting (WAP). Weeding was done manually 3 and 6 WAP. A recommended rates of $140 \mathrm{~kg} \mathrm{~N}^{-1}$ and $70 \mathrm{~kg} \mathrm{~N}^{-1}$ for hybrid and open pollinated maize respectively were applied in two splits at planting in form of NPK 15- 1515 and urea fertilizer at 4 weeks after planting as top dressing (Aduayi et al., 2002). Defoliation of two uppermost leaves was conducted 4 days after silking (DAS) as described by Liu et al., (2015).

\subsection{Sampling and data collection}

Soil samples were randomly collected from the sites before planting for the determination of pre-planting soil physico-chemical properties. Agronomic, phenological, physiological as well as yield and yield component variables were taken at 4WAP (vegetative stage), 8WAP (reproductive stage) and 12WAP (physiological maturity stage). Five plants were tagged randomly from the net plot. Plant height was determined from the soil surface to the tip of the last formed leaf. Leaf area was evaluated using the formula Leaf Area = length $\times$ widest width $\times 0.75$. The correction factor was 0.75 (Dwyer and Stewart, 1986) and leaf area index was calculated as the ratio of total leaf area to land area: $\frac{\text { Leafarea }\left(\mathrm{cm}^{2}\right)}{\text { Landarea }\left(\mathrm{cm}^{2}\right)}$

Phenological variables (days to $50 \%$ anthesis, and days to $50 \%$ silking) were determined by standard procedures. Yield and yield component variables were determined by standard agronomic procedures at harvest maturity.Above the ground biomass was sampled at 4DAS (days after silking) and R6 (physiological maturity). These was later packed in an envelope and placed in an electric oven at $60^{\circ} \mathrm{C}$ until constant mass was obtained. The samples were removed, cooled for about 30 minutes and weighed. Post-silking source-sink ratio(PSSR) was determined as the change in aboveground biomass dry massduring 4 DAS and at physiological maturity.Dry matter translocation (DMT) (rate of remobilization) $\left(\mathrm{kg} \mathrm{ha}^{-1}\right)=$ dry matter at anthesis - dry mass at maturity (all vegetative parts except grains). While dry matter translocation efficiency (DMTE) (Remobilization Efficiency) $(\%)=$ dry matter translocation / dry matter at anthesis $\times 100$.Rate of current photosynthesis (RCP) was calculated using the formula: grain yield $\left(\mathrm{kg} \mathrm{ha}^{-1}\right)$ - dry matter translocation $\left(\mathrm{kg} \mathrm{ha}{ }^{-1}\right)$ and efficiency of current photosynthesis (ECP): rate of current photosynthesis $\left(\mathrm{kg} \mathrm{ha}^{-1}\right) /$ dry massof vegetative organs at maturity stage $\left(\mathrm{kg} \mathrm{ha}^{-1}\right)$ (Papakosta and Gayianas, 1991; Van Sanford and Mackown, 1987).

\subsection{Statistical analysis}

Data collected were subjected to mixed model analysis of variance (ANOVA), at $5 \%$ probability level and means of significant treatmentwere separated using least significant difference (LSD). The statistical package used was Genstat $12^{\text {th }}$ Edition. 


\section{RESULTS}

At Abeokuta maximum amount of rainfall $(165 \mathrm{~mm})$ during the cropping season was observed in June, 2015, while no precipitation was recorded in January of that year. Similar pattern was observed on relative humidity, except that the maximum relative humidity was observed in July. Temperature was in the range of $30.2{ }^{\circ} \mathrm{C}$ and $26.8^{\circ} \mathrm{C}$ for the months of March and June respectively (Table 1). Ibadan had similar rainfall pattern as Abeokuta except for the quantity of rainfall observed for the months during the cropping season.
Maximum amount of rainfall $(321.9 \mathrm{~mm})$ was observed in May with the minimum $(6.6 \mathrm{~mm})$ recorded in January. Consequently higher relative humidity was observed in Ibadan ranged between $92 \%$ to $88 \%$ in August and February respectively. However, February and March had similar relative humidity during the cropping season. The highest temperature $\left(29^{\circ} \mathrm{C}\right)$ was observed in February while the least $\left(25^{\circ} \mathrm{C}\right)$ was observed in August (Table 2).

Table 1: Means of agrometeorological observations, Abeokuta

\begin{tabular}{lccc}
\hline Months & Relative & Humidity & \\
$(\%)$ & Mean temperature $\left({ }^{\circ} \mathrm{C}\right)$ \\
\hline January & Rainfall $(\mathrm{mm})$ & 47.7 & 27.8 \\
February & 0.0 & 61.4 & 29.5 \\
March & 51.0 & 60.4 & 30.2 \\
April & 67.0 & 62.8 & 29.0 \\
May & 69.0 & 61.9 & 28.3 \\
June & 60.0 & 70.8 & 26.8 \\
July & 165.0 & 73.0 & 27.2 \\
August & 66.0 & 70.3 & 26.2 \\
\hline Source: Department of Agrometeorological and Water Management, Federal University of Agriculture, Abeokuta.
\end{tabular}

Table 2: Means of agrometeorological observations, Ibadan

\begin{tabular}{lccc}
\multicolumn{1}{c}{ Months } & Rainfall $(\mathrm{mm})$ & Relative Humidity $(\%)$ & Mean temperature $\left({ }^{\circ} \mathrm{C}\right)$ \\
\hline January & 6.6 & 90.0 & 28.5 \\
February & 28.4 & 88.0 & 29.0 \\
March & 189.6 & 88.0 & 28.0 \\
April & 246.3 & 89.0 & 28.5 \\
May & 321.9 & 89.0 & 28.0 \\
June & 233.7 & 90.0 & 28.5 \\
July & 157.9 & 82.0 & 26.0 \\
August & 139.4 & 92.0 & 25.0
\end{tabular}

Source: Weather station, National Horticultural Institute of Nigeria (NIHORT).

\subsection{Growth response}

Plant height was similar between the maize cultivarsat each period of investigation in each location(Tables 3 and 4). There was no significant $(P>0.05)$ varietal variability on leaf area and leaf area index at Abeokuta, except at 4 WAP. Variety 2009 TZE-W DT STR had larger leaf area and leaf area index than TZEI $124 \mathrm{X}$ TZEI 25.Leaf area index significantly $(P<0.05)$ increased as plant population increases across all periods of investigation at both locations (Tables 3 and 4).

At 12WAP defoliated maize cultivar was significantly shorter than non-defoliated at Abeokuta (Table 3). 
Table 3: Effect of plant population and post-anthesis defoliation on the means of growth of maize variables at 4, 8 and $12 \mathrm{WAP}$, Abeokuta

\begin{tabular}{|c|c|c|c|c|c|c|c|c|c|}
\hline Treatments & \multicolumn{3}{|c|}{ Plant height $(\mathrm{cm})$} & \multicolumn{3}{|c|}{ Leaf area $\left(\mathrm{cm}^{2}\right)$} & \multicolumn{3}{|c|}{ Leaf area index } \\
\hline Variety (V df 1) & $4 \mathrm{WAP}$ & $8 \mathrm{WAP}$ & $12 \mathrm{WAP}$ & 4 WAP & $8 \mathrm{WAP}$ & $12 \mathrm{WAP}$ & 4 WAP & $8 \mathrm{WAP}$ & 12 WAP \\
\hline TZEI $124 \times$ TZEI 25 & 116.57 & 199.74 & 209.52 & 392.29 & 635.3 & 642.22 & 0.13 & 0.21 & 0.21 \\
\hline 2009 TZE-W DT STR & 119.15 & 202.68 & 217 & 457.94 & 660.13 & 675.18 & 0.15 & 0.22 & 0.22 \\
\hline $\operatorname{LSD}(5 \%)$ & NS & NS & NS & $43.12 *$ & NS & NS & $0.01 *$ & NS & NS \\
\hline \multicolumn{10}{|c|}{ Plant Population (PP df 2) } \\
\hline 106666 plants ha ${ }^{-1}$ & 126.41 & 206.03 & 218.42 & 456.71 & 671.79 & 669.24 & 0.24 & 0.36 & 0.36 \\
\hline 80000 plants ha ${ }^{-1}$ & 116.58 & 198.33 & 213.17 & 434.44 & 640.97 & 658.98 & 0.12 & 0.18 & 0.17 \\
\hline 71111 plants ha ${ }^{-1}$ & 110.58 & 199.28 & 208.20 & 384.19 & 630.37 & 647.87 & 0.07 & 0.12 & 0.11 \\
\hline $\operatorname{LSD}(5 \%)$ & NS & NS & NS & NS & NS & NS & $0.03 * *$ & $0.02 * *$ & $0.03 * *$ \\
\hline \multicolumn{10}{|l|}{ Defoliation (D df 1) } \\
\hline No Defoliation & & 203.48 & 217.91 & & 649.94 & 664.20 & & 0.22 & 0.21 \\
\hline Defoliated & & 198.94 & 208.61 & & 645.49 & 653.19 & & 0.21 & 0.21 \\
\hline $\operatorname{LSD}(5 \%)$ & & NS & $8.63 *$ & & NS & NS & & NS & NS \\
\hline$V \times P P($ df 2$)$ & & NS & NS & & NS & NS & & NS & NS \\
\hline $\mathrm{V} \times \mathrm{D}(\mathrm{df} 1)$ & & NS & NS & & NS & NS & & NS & NS \\
\hline$P P \times D($ df 2$)$ & & NS & NS & & NS & NS & & NS & NS \\
\hline $\mathrm{V} \times \mathrm{D} \times \mathrm{PP}(\mathrm{df} 2)$ & & NS & NS & & NS & NS & & NS & NS \\
\hline
\end{tabular}

\footnotetext{
$*$ indicates significance at $P<0.05$ probability level. WAP: Weeks after planting, NS: Not significant, df- degree of freedom
} 
Olalekan SAKARIYAWO et al.

Table 4: Effect of plant population and post-anthesis defoliation on the means of growth variables of maize varieties at 4,8 and 12 WAP, Ibadan

\begin{tabular}{|c|c|c|c|c|c|c|c|c|c|}
\hline \multirow{2}{*}{$\begin{array}{ll}\text { Treatments } \\
\text { Variety (V df 1) }\end{array}$} & \multicolumn{3}{|c|}{ Plant height $(\mathrm{cm})$} & \multicolumn{3}{|c|}{ Leaf area $\left(\mathrm{cm}^{2}\right)$} & \multicolumn{3}{|c|}{ Leaf area index } \\
\hline & 4 WAP & $8 \mathrm{WAP}$ & $12 \mathrm{WAP}$ & 4 WAP & $8 \mathrm{WAP}$ & $12 \mathrm{WAP}$ & $4 \mathrm{WAP}$ & $8 \mathrm{WAP}$ & $12 \mathrm{WAP}$ \\
\hline TZEI $124 \times$ TZEI 25 & 72.44 & 142 & 159.56 & 173.96 & 491.5 & 445.64 & 0.06 & 0.16 & 0.15 \\
\hline 2009 TZE-W DT STR & 71.29 & 147.28 & 171 & 188.23 & 501.65 & 474.04 & 0.06 & 0.16 & 0.16 \\
\hline $\operatorname{LSD}(5 \%)$ & NS & NS & NS & NS & NS & NS & NS & NS & NS \\
\hline \multicolumn{10}{|l|}{ Plant Population (PP df 2) } \\
\hline 106666 plants ha ${ }^{-1}$ & 72.68 & 150.26 & 170.69 & 202.78 & 468.78 & 493.06 & 0.11 & 0.25 & 0.26 \\
\hline 80000 plants ha ${ }^{-1}$ & 70.93 & 144.5 & 165.97 & 167.07 & 499.76 & 453.59 & 0.04 & 0.13 & 0.12 \\
\hline 71111 plants ha ${ }^{-1}$ & 71.99 & 139.17 & 159.18 & 173.44 & 521.18 & 432.86 & 0.03 & 0.1 & 0.08 \\
\hline $\operatorname{LSD}(5 \%)$ & NS & NS & NS & NS & NS & NS & $0.01 * *$ & $0.02 * *$ & $0.01^{* *}$ \\
\hline \multicolumn{10}{|l|}{ Defoliation (D df 1) } \\
\hline No Defoliation & & 143.36 & 164.22 & & 493.67 & 467.77 & & 0.17 & 0.16 \\
\hline Defoliated & & 145.92 & 166.34 & & 499.47 & 451.91 & & 0.16 & 0.15 \\
\hline $\operatorname{LSD}(5 \%)$ & & NS & NS & & NS & NS & & NS & NS \\
\hline$V \times P P($ df 2$)$ & & NS & NS & & NS & NS & & NS & NS \\
\hline $\mathrm{V} \times \mathrm{D}(\mathrm{df} 1)$ & & NS & NS & & NS & NS & & NS & NS \\
\hline $\mathrm{PP} \times \mathrm{D}(\mathrm{df} 2)$ & & NS & NS & & NS & NS & & NS & NS \\
\hline $\mathrm{V} \times \mathrm{D} \times \mathrm{PP}(\mathrm{df} 2)$ & & NS & NS & & $90.59 *$ & NS & & NS & NS \\
\hline
\end{tabular}


Physiological and agronomic responses of maize $($ Zea mays L) ... and defoliation at post-anthesis in the humid rainforest

\subsection{Dry matter translocation, current photosynthesis, phenology, yield and yield components}

At both locations there were no significant varietal differences on translocation and post silking source sink variables except on dry matter translocation efficiency at Ibadan (Table 5). Dry matter was more efficiently remobilised in maize variety TZEI $124 \times$ TZEI 25 than variety 2009 TZE-W DT STR. With increase plant population dry matter was more remobilised in plant population of 80000 plants $\mathrm{ha}^{-1}$.Thereafter, a significant depression was observed at Ibadan. Defoliation had no significant effect on the aforementioned variables at both locations (Table 5). Significant $(P<0.05)$ varietal variability was observed on days to $50 \%$ anthesis at both locations (Tables 6 and 7). Variety 2009 TZE-W DT STR attained $50 \%$ anthesis earlier than variety TZEI $124 \times$ TZEI 25 .Significant $(P<0.05)$ varietal variability was observed on rate of current photosynthesis at Abeokuta (Table 6). Maize variety 2009 TZE-W DT STR had significantly higher rate of current photosynthesis than maize variety TZEI $124 \times$ TZEI 25.Plant population had significant $(P<0.05)$ effecton rate of current photosynthesis at Abeokuta and Ibadan. Plant population of 80000 plants ha $^{-1}$ and 71111 plants $\mathrm{ha}^{-1}$ had the highest rate of current photosynthesis while plant population of 106666 plants $^{-1} \mathrm{a}^{-1}$ and 80000 had significantly the least rate of current photosynthesis at Abeokuta and Ibadan respectively.Increasing plant population significantly $(P<0.05)$ decreased number of kernels per row at Abeokuta (Table 6). Significant varietal variability was observed in number of kernels per row at Ibadan where variety TZEI $124 \times$ TZEI 25 had significantly higher kernels per row than 2009 TZEW DT STR (Table 7). Significant varietal difference $(P$ $<0.05)$ was observed on one thousand grain massat Abeokuta (Table 6). Heavier one thousand grain masswas found in variety 2009 TZE-W DT STR compared with variety TZEI $124 \times$ TZEI 25 .Defoliation had significant effect on $(P<0.05)$ on one thousand grain mass across the locations. Non-defoliated maize produced heavier 1000 grain massthan defoliated maize. Grain yield differ significantly $(P<0.05)$ among plant populations at Ibadan (Table 7). The order of increase in grain yield obtained from plant population treatments was 106666 plants $\mathrm{ha}^{-1}>71111$ plants $\mathrm{ha}^{-1}>80000$ plants ha ${ }^{-1}$. 
Olalekan SAKARIYAWO et al.

Table 5: Effect of plant population and post -anthesis defoliation on the means of physiological variables of maize varieties (Abeokuta and Ibadan)

\begin{tabular}{|c|c|c|c|c|c|c|}
\hline \multirow{3}{*}{ Treatments } & \multicolumn{3}{|c|}{ Abeokuta } & \multicolumn{3}{|c|}{ Ibadan } \\
\hline & \multicolumn{3}{|c|}{ Translocation variables } & \multicolumn{3}{|c|}{ Translocation variables } \\
\hline & DMT $\left(\mathrm{kg} \mathrm{ha}^{-1}\right)$ & $\operatorname{DMTE}(\%)$ & PSSSR & DMT $\left(\mathrm{kg} \mathrm{ha}^{-1}\right)$ & DMTE $(\%)$ & PSSSR \\
\hline \multicolumn{7}{|l|}{ Variety (V df 1) } \\
\hline TZEI $124 \times$ TZEI 25 & 2847.09 & 9.26 & -0.12 & 3028.59 & 6.35 & -23.93 \\
\hline 2009 TZE-W DT STR & 783.79 & -4.22 & 0.38 & 153.98 & -22.38 & -21.86 \\
\hline $\operatorname{LSD}(5 \%)$ & NS & NS & NS & NS & $50.68 *$ & NS \\
\hline \multicolumn{7}{|c|}{ Plant population (PP df 2) } \\
\hline 106666 plants ha ${ }^{-1}$ & 3426.91 & 6.21 & -0.05 & 3530.97 & 13.08 & -29.95 \\
\hline 80000 plants ha ${ }^{-1}$ & 638.3 & -2.91 & 0.3 & 3711.13 & 23.5 & -17.87 \\
\hline 71111 plants ha ${ }^{-1}$ & 1381.11 & 4.26 & 0.13 & -2468.26 & -60.62 & -20.86 \\
\hline $\operatorname{LSD}(5 \%)$ & NS & NS & NS & $2952.95 *$ & NS & NS \\
\hline \multicolumn{7}{|l|}{ Defoliation (D df 1) } \\
\hline No Defoliation & 3190.91 & 10.34 & -0.28 & 1138.99 & -22.25 & -22.19 \\
\hline Defoliated & 439.96 & -5.3 & 0.53 & 2043.57 & 6.21 & -23.6 \\
\hline $\operatorname{LSD}(5 \%)$ & NS & NS & NS & NS & NS & NS \\
\hline$V \times P P($ df 2$)$ & $9299.98 *$ & NS & NS & NS & NS & NS \\
\hline $\mathrm{V} \times \mathrm{D}(\mathrm{df} 1)$ & NS & NS & NS & NS & NS & NS \\
\hline $\mathrm{PP} \times \mathrm{D}(\mathrm{df} 2)$ & NS & NS & NS & NS & $61.54 *$ & NS \\
\hline $\mathrm{V} \times \mathrm{D} \times \mathrm{PP}(\mathrm{df} 2)$ & NS & NS & NS & NS & NS & NS \\
\hline
\end{tabular}

* indicates significance at $\mathrm{P}<0.05$ probability level and ** indicates significance at $\mathrm{P}<0.01$ probability level. DMT: Dry matter translocation, DMTE: Dry matter translocation efficiency, PSSSR: Post silking source sink ratio, df: degree of freedom 
Table 6: Effect of plant population and post-anthesis defoliation on the means of phenology, current photosynthesis, yield and yield components of maize varieties, Abeokuta

\begin{tabular}{|c|c|c|c|c|c|c|c|}
\hline Treatments & $\begin{array}{c}50 \% \\
\text { Anthesis }\end{array}$ & $\begin{array}{l}50 \% \\
\text { Silking }\end{array}$ & $\mathrm{ECP} \mathrm{kg} \mathrm{kg}_{1}^{-}$ & $\mathrm{RCP}_{1}^{\mathrm{kg} \text { ha }}$ & $\begin{array}{c}\text { Number of kernels per } \\
\text { row }\end{array}$ & $\begin{array}{c}1000 \text { grain mass } \\
(\mathrm{g})\end{array}$ & Grain mass $\mathrm{kg}_{1}^{-}$ \\
\hline \multicolumn{8}{|l|}{ Variety (V df 1) } \\
\hline TZEI $124 \times$ TZEI 25 & 44.89 & 47.72 & 0.17 & 2222.25 & 30.47 & 146.67 & 5069.33 \\
\hline 2009 TZE-W DT STR & 44.00 & 47.33 & 0.33 & 5014.22 & 27.04 & 186.06 & 5798.01 \\
\hline $\operatorname{LSD}(5 \%)$ & $0.632 *$ & NS & NS & $14058.51 *$ & NS & $14.722 * *$ & NS \\
\hline \multicolumn{8}{|c|}{ Plant Population (PP df 2) } \\
\hline 106666 plants ha $^{-1}$ & 45.17 & 48.17 & 0.15 & 1947.58 & 25.73 & 164.33 & 5374.49 \\
\hline 80000 plants ha $^{-1}$ & 44.17 & 47.17 & 0.35 & 5092.83 & 29.92 & 174.67 & 5731.13 \\
\hline 71111 plants ha ${ }^{-1}$ & 44 & 47.25 & 0.24 & 3814.29 & 30.6 & 160.08 & 5195.4 \\
\hline $\operatorname{LSD}(5 \%)$ & NS & NS & NS & $2077.45^{*}$ & $2.604 * *$ & NS & NS \\
\hline \multicolumn{8}{|l|}{ Defoliation (D df 1) } \\
\hline No Defoliation & 44.33 & 47.61 & 0.16 & 2455.69 & 28.24 & 173.78 & 5646.6 \\
\hline Defoliated & 44.56 & 47.44 & 0.33 & 4780.77 & 29.26 & 158.94 & 5220.74 \\
\hline $\operatorname{LSD}(5 \%)$ & NS & NS & NS & NS & NS & $10.377 * *$ & NS \\
\hline $\mathrm{V} \times \mathrm{PP}(\mathrm{df} 2)$ & NS & NS & $0.72 *$ & $12553.18^{* *}$ & $4.299^{*}$ & NS & NS \\
\hline $\mathrm{V} \times \mathrm{D}(\mathrm{df} 1)$ & NS & NS & NS & NS & NS & NS & NS \\
\hline $\mathrm{PP} \times \mathrm{D}(\mathrm{df} 2)$ & NS & NS & NS & NS & NS & NS & NS \\
\hline $\mathrm{V} \times \mathrm{D} \times \mathrm{PP}(\mathrm{df} 2)$ & NS & NS & NS & NS & NS & NS & NS \\
\hline
\end{tabular}


Table 7: Effect of plant population and post-anthesis defoliation on the means of phenology, current photosynthesis, yield and yield components of maize varieties, Ibadan

\begin{tabular}{|c|c|c|c|c|c|c|c|}
\hline Treatments & $\begin{array}{c}50 \% \\
\text { Anthesis }\end{array}$ & $\begin{array}{l}50 \% \\
\text { Silking } \\
\end{array}$ & $\begin{array}{c}\mathrm{ECP} \mathrm{kg} \\
\mathrm{kg}^{-1}\end{array}$ & RCP kg ha ${ }^{-1}$ & $\begin{array}{c}\text { Number of kernels per } \\
\text { row }\end{array}$ & $\begin{array}{c}1000 \text { grain mass } \\
(\mathrm{g})\end{array}$ & $\begin{array}{c}\text { Grain mass kई } \\
\mathrm{ha}^{-1}\end{array}$ \\
\hline \multicolumn{8}{|l|}{ Variety (V df 1) } \\
\hline TZEI 124 X TZEI 25 & 49.78 & 53.61 & 0.44 & 2446.54 & 27.63 & 150.44 & 5475.13 \\
\hline 2009 TZE-W DT STR & 48.94 & 53.33 & 0.75 & 4815.39 & 22.29 & 166.78 & 4969.36 \\
\hline $\operatorname{LSD}(5 \%)$ & $0.82 *$ & NS & NS & NS & $4.033^{*}$ & NS & NS \\
\hline \multicolumn{8}{|l|}{ Plant Population (PP df 2) } \\
\hline 106666 plants ha $^{-1}$ & 50.00 & 54.08 & 0.31 & 2592.73 & 22.69 & 152.17 & 6123.70 \\
\hline 80000 plants ha $^{-1}$ & 49.08 & 53.17 & 0.13 & 590.94 & 26.10 & 163.58 & 4302.08 \\
\hline 71111 plants ha ${ }^{-1}$ & 49.00 & 53.17 & 1.35 & 7709.23 & 26.09 & 160.08 & 5240.97 \\
\hline $\operatorname{LSD}(5 \%)$ & NS & NS & $0.77^{*}$ & $3206.01 * *$ & NS & NS & $1364.50 *$ \\
\hline \multicolumn{8}{|l|}{ Defoliation (D df 1) } \\
\hline No Defoliation & 49.33 & 53.56 & 0.80 & 3944.68 & 24.34 & 169.67 & 5083.67 \\
\hline Defoliated & 49.39 & 53.39 & 0.39 & 3317.25 & 25.58 & 147.56 & 5360.82 \\
\hline $\operatorname{LSD}(5 \%)$ & NS & NS & NS & NS & NS & $11.59^{* *}$ & NS \\
\hline$V \times P P(d f 2)$ & NS & NS & NS & NS & NS & $19.80^{*}$ & NS \\
\hline $\mathrm{V} \times \mathrm{D}(\mathrm{df} 1)$ & NS & NS & NS & NS & NS & NS & NS \\
\hline$P P \times D(d f 2)$ & NS & NS & $0.88^{*}$ & NS & NS & NS & NS \\
\hline $\mathrm{V} \times \mathrm{D} \times \mathrm{PP}(\mathrm{df} 2)$ & NS & NS & NS & $12101.19 *$ & NS & NS & NS \\
\hline
\end{tabular}




\section{DISCUSSION}

The open pollinated maize variety (2009 TZE-W DT STR ) displayed better growth variables than the hybrid maize (TZEI $124 \times$ TZEI 25) at Abeokuta. The higher leaf area and leaf area index could increase the assimilatory surface of this variety for the interception of light and increased transpiration. These facts combined could have aided the photosynthetic capacity of OPV as reflected in the significantly higher rate of current photosynthesis in this variety than the hybrid maize at Abeokuta. Reduced dry matter translocation efficiency at Ibadan suggested that there could be enough assimilate available to sustain the growth of OPV. Availability of sufficient assimilate at Ibadan could be linked to precipitation pattern observed during the cropping season. It had earlier been reported that increasing dry matter translocation efficiency could predispose crops to increased rate of leaf senescence especially under nitrogen deficiency (He et al., 2003), subsequently compromising crop performance. Shorter days to $50 \%$ anthesis could have increased duration of reproductive structures in the absence of production constraints. This was reflected in the significantly higher 1000 grain mass observed in OPV than the maize hybrid at Abeokuta. However, in Abeokuta despite the presence of more macronutrients than Ibadan rainfall distribution could constraint their availability. Water plays a significant role in nutrient availability, especially the solubility of mobile nutrients and their movement to the rhizosphere. This environmental constraint could have suggested that OPV could not optimally utilise the available growth resources. A shorter phenology could have enabled OPV to escape this production constraint. As a consequence this could have affected its performance, which was not significantly different from that of the hybrid maize at both locations despite the morphological and physiological advantages earlier displayed. The significant depression in the number of kernels per row in Ibadan might be linked with the reduced dry matter translocation efficiency observed in OPV than hybrid maize. This pattern would have a profound effect on the grain yield of OPV at Ibadan if current photosynthesis was not adequate enough to sustain assimilate availability at the grain filling growth stage. However, the rate of current photosynthesis and 1000 grain mass for this variety was higher than the hybrid maize though not significant at Ibadan. This could have implied a kind of compensatory relationship between the grain mass and number kernel. Such relationship had been reported in the cereals in the past, especially under stressful condition (Squire, 1990).

Increased plant population at both locations resulted in increased LAI at all periods of investigation. This increased assimilatory surface could predispose maize variety to increased interception of light and transpiration especially under vapour pressure deficit. Increased transpiration could have been more pronounced at Abeokuta, where there was higher temperature with reduced relative humidity than Ibadan. With the rainfall pattern observed at Abeokuta there could be possibility of subjecting maize variety to soil moisture deficit. This response could compromise photosynthetic rate. However, the pattern of RCP at both locations with increasing plant population indicated that maize variety at Abeokuta was able to acclimatise to increasing plant population than those at Ibadan, though they both displayed depression in RCP albeit at different plant populations. That could be partly explained by the distribution of nitrogen in the canopy. High plant population had been reported to have resulted in increased light attenuation and distribution of nitrogen along the strata vertically (Maddonni et al., 2001). This pattern could be further accentuated by deficiency of nitrogen in the soil thereby compromising canopy photosynthetic capacity through increased senescence that could have resulted from increased remobilisation of assimilates from other organs in maize plant. However the evidences available at Ibadan supported the hypothesis of increased remobilisation of assimilates with high plant population as indicated in the increased dry matter translocation when maize population attained population of 80000 plants ha ${ }^{-1}$ with converse pattern on rate of current photosynthesis and efficiency of current photosynthesis. Senescence could further be simulated by reduced red to far red ratio observed with increasing light attenuation in the canopy at both locations (Varlet-Grancher and Gautier, 1995). At Abeokuta the depression observed in the number of kernels per row with increasing plant population could have been linked to the assimilate availability. Increasing plant population at this location reduced RCP which could have compromised assimilate availability. This observation was corroborated by Tollenaar and Daynard, (1982). Maize plant had been observed to have reduced remobilisation efficiency in post-anthesis source-sink manipulation. The remobilisation efficiency of maize was evaluated to be $0.26 \mathrm{~g}$ of seed $\mathrm{g}^{-1}$ of stored carbohydrate, which was significantly lower than that of wheat and soybean (Kiniry et al., 1992). At Ibadan increased grain yield with increasing plant population would have been explained by the response pattern observed in RCP and efficiency of current photosynthesis. Carbon supply through the increased photosynthetic variables observed at high plant population at Ibadan especially at the critical growth stage of maize could increase nitrogen uptake by the root. This is even more germane at Ibadan where soil nitrogen content is lower than Abeokuta. But with increased root growth and higher precipitation the 
possibility of mining this nutrient is very high. Uhart and Andrade, (1995) had earlier posited that availability of assimilates at the grain filling period could increase root growth and possibly increase the grain yield. This observation was further corroborated by Moll et al.(1994).

At both locations reduced 1000 grain mass could have been responsible for the performance of defoliated maize at post anthesis. However there were no significant differencesin the performances of defoliated and non-defoliated maize at both locations. This could have suggested that other yield components could have contributed to the performance of defoliated and nondefoliated maize apart from the number of reproductive structures. It had earlier been posited that if a significant effect in the alternation of source sink ratio was observed on the seed dry mass it could be as a result of source limitation (Borrás et al., 2004). This could be linked to the change in canopy architecture through a change in plant height as observed in reduced plant height in defoliated maize crop compared to the nondefoliated at post anthesis period in Abeokuta. This reduced plant height could limit the penetration of both direct and diffuse light into the canopy. Reduced light into the canopy could compromise canopy photosynthesis and subsequently availability of the assimilates to the reproductive structures, especially at the grain filling stage. Maize had been reported to change seed mass to assimilate availability more than wheat especially under high solar radiation and temperature during the seed filling period, however it is highly sensitive under reduced assimilate availability
(Borrás et al., 2004). The limited availability of assimilates due to source restriction would compromise the grain yield in maize if considered in the context of comparatively reduced remobilisation efficiency of maize compared to other grain legumes and cereals (Kiniry et al., 1992). This observation is in contraction of the results obtained by Liu et al., (2015). In their trial defoliation resulted in increased grain yield. The increased grain yield under defoliation was premised on higher canopy apparent photosynthesis, reduced senescence and reduced source:sink ratio than nondefoliated maize crop. Other observations that contributed to increased grain yield in defoliated maize crop than non-defoliated were increased harvest index, increased kernel mass and ear mass in their trial. It could be suggested that the high plant population used in their trial within the context of prevailing temperature in their condition resulted in sink limitation of maize crop performance. It had been reported that the performance of maize could be both source or sink limited (Thomas, 1992). This limitation is based on the threshold of assimilate availability, above or below which the performance of maize crop could be compromised. The differences in the genetic make-up of the varieties used in both experiments could have accounted for the differences in the results obtained. Same argument could be used when considering the environment and the management techniques in place when both trials were carried out, which were considerably different. However, the underlying mechanism that underpins differences in physiological and agronomic responses in both trials needs further research.

\section{CONCLUSION}

The investigation demonstrated that varietal differences observed between the maize varieties was reflected in their growth, phenology and assimilate translocation variables. The significantly higher assimilatory surface of OPV than the hybrid maize could have supported the interception of more radiant energy and gas exchange processes as indicated in rate of current photosynthesis, reduced dry matter translocation efficiency and bigger 1000 grain mass. However, the prevailing rainfall pattern at Abeokuta could have compromised the positive physiological responses of OPV. Thus the reduced days to $50 \%$ anthesis observed could have reduced the potential utilisation of growth factors to attain yield potential. These evidences taken together would have suggested that OPV could attain similar grain yield to the hybrid under these agroecological conditions . This could be of economic benefit taken into consideration the cost implication of obtaining hybrid seed especially among resource challenged farmers. Both locations experienced increased LAI with increased plant population. However, response pattern of rate of current photosynthesis differs with increasing plant population at both locations. At Ibadan at population of 80000 plants $\mathrm{ha}^{-1}$ maize variety experienced reduced rate of current photosynthesis and efficiency of current photosynthesis with concomitant increase in high dry matter translocation probably to support grain set and grain filling as reflected in high grain yield at plant density of 106666 plant ha ${ }^{-1}$. Contrarily, at Abeokuta the highest rate of current photosynthesis was attained at the plant density of 80000 plant $\mathrm{ha}^{-1}$. This response could have made assimilate available to support reproductive structures as indicated in the grain yield at that plant population. Further increase in the plant population could have resulted in mutual shading in the canopy. These discrepancies in the response pattern to plant population could have been explained by the differences in the intensity of rainfall and its effect on nutrient availability especially at Abeokuta that experienced reduced 
intensity of rainfall. Reduced 1000 grain mass at both locations when maize varieties were defoliation suggested a disruption in source:sink balance through source constraint. This was validated at Abeokuta with a decrease in plant height that could have affected maize canopy architecture, though maize had similar grain yield at both locations under this treatment. It could be concluded that defoliation of two uppermost leaves had no significant effect on the performance of maize varieties in this agroecology.

\section{ACKNOWLEDGMENTS}

We would like to extend our profound appreciation to Dr Akpraku, a Scientist with IITA for providing the planting materials. Similar gesture goes to Dr Ajayi at the National Horticultural Institute of Nigeria (NIHORT), Ibadan for his efforts in the establishment of field trial at Ibadan. Dr M.O. Dare and Dr A.A. Oyekanmi of the Department of Soil Science and Land Management and Plant Physiology and Crop Production respectively are highly appreciated for the initial review of this manuscript.

\section{REFERENCES}

Aduayi, E., Chude, V., Adebusuyi, B., Olayiwola, S. (2002). Fertiliser use and management practises for crop in Nigeria (3rd ed.). Abuja: Federal Fertiliser Department, Federal Ministry of Agriculture and Rural Development.

Ahmadi, A., Joudi, M. (2007). Effects of timing and defoliation intensity on growth, yield and gas exchange rate of wheat grown under well-watered and drought conditions. Pakistan Journal of Biology Science, 10(21), 3794-3800. doi:10.3923/pjbs.2007.3794.3800

Allison, L. (1965). Organic carbon. In: C.A Black (Ed.), Methods of soil analysis. Part 2 (pp. 1307-1378). Madison: American Society of Agronomy.

Borrás, L., Maddonni, G. A., Otegui, M. E. (2003). Leaf senescence in maize hybrids: plant population, row spacing and kernel set effects. Field Crops Research, 82(1), 13-26. doi:10.1016/S03784290(03)00002-9

Borrás, L., Slafer, G. A., Otegui, M. E. (2004). Seed dry weight response to source-sink manipulations in wheat, maize and soybean: a quantitative reappraisal. Field Crops Research, 86(2), 131-146. doi:10.1016/j.fcr.2003.08.002

Bouyoucos, G. (1962). Hydrometer method improved for making particle size analysis of soil. Agronomy Journal, 54, 464-465. doi:10.2134/agronj1962.00021962005400050028x

Bray, R., Kurtz, L. (1945). Determination of total, organic and available forms of phosphorus in soil. Soil Science, 59, 39-45. doi:10.1097/00010694194501000-00006

Dwyer, L.,Stewart, D. (1986). Leaf area development in field grown maize. Agronomy Journal, 78, 334-
343. doi:10.2134/agronj1986.00021962007800020024x

Gonzalo, M., Vyn, T. J., Holland, J. B., McIntyre, L. M. (2006). Mapping density response in maize: a direct approach for testing genotype and treatment interactions. Genetics, 173(1), 331-348. doi:10.1534/genetics.105.045757

He, P., Osaki, M., Takebe, M., Shinano, T. (2003). Comparison of whole system of carbon and nitrogen accumulation between two maize hybrids differing in leaf senescence. Photosynthetica, 41(3), 399-405.

doi:10.1023/B:PHOT.0000015464.27370.60

Jackson, M. (1962). Soil chemical analysis. New Delhi: Prentice Hall of India Pvt, Ltd.

Kiniry, J. R., Tischler, C. R., Rosenthal, W. D., Gerik, T. J. (1992). Nonstructural carbohydrate utilization by sorghum and maize shaded during grain growth. Crop Science, 32(1), 131-137. doi:10.2135/cropsci1992.0011183X003200010029 $\mathrm{x}$

Lemcoff, J. H., Loomis, R. S. (1994). Nitrogen and density influences on silk emergence, endosperm development, and grain yield in maize (Zea mays L.). Field Crops Research, 38(2), 63-72. doi:10.1016/0378-4290(94)90001-9

Liu, T., Gu, L., Dong, S., Zhang, J., Liu, P., Zhao, B. (2015). Optimum leaf removal increases canopy apparent photosynthesis, 13 C-photosynthate distribution and grain yield of maize crops grown at high density. Field Crops Research, 170, 32-39. doi:10.1016/j.fcr.2014.09.015

Maddonni, G. A., Otegui, M. E., Cirilo, A. G.( 2001). Plant population density, row spacing and hybrid effects on maize canopy architecture and light 
attenuation. Field Crops Research, 71(3), 183-193. doi:10.1016/S0378-4290(01)00158-7

Martin, R. V., Washington, R., Downing, T. E. (2000). Seasonal maize forecasting for South Africa and Zimbabwe derived from an agroclimatological model. Journal of Applied Meteorology, 39(9), 1473-1479. doi:10.1175/15200450(2000)039<1473:SMFFSA>2.0.CO;2

McLean, E. O. (1982). Soil $\mathrm{pH}$ and lime requirement. Methods of Soil Analysis. Part 2. Chemical and Microbiological Properties, (methodsofsoilan2), 199-224.

Moll, R. H., Jackson, W. A., Mikkelsen, R. L. (1994). Recurrent selection for maize grain yield: dry matter and nitrogen accumulation and partitioning changes. Crop Science, 34(4). 874-881. doi:10.2135/cropsci1994.0011183X003400040009 $\mathrm{x}$

Murphy, J., Riley, J. P. (1962). A Modified Single Solution Method for Determination of Phosphate in Natural Waters. Analytica Chimica Acta, 27, 3136. doi:10.1016/S0003-2670(00)88444-5

Papakosta, D., Gayianas, A. (1991). Nitrogen and dry matter accumulation, remobilisation and losses for Mediterranean wheat during grain filling. Agronomy Journal, 83, 864-870. doi:10.2134/agronj1991.00021962008300050018x

Rajcan, I., Tollenaar, M. (1999). Source: sink ratio and leaf senescence in maize: I. Dry matter accumulation and partitioning during grain filling. Field Crops Research, 60(3), 245-253. doi:10.1016/S0378-4290(98)00142-7

Sangoi, L. (2001). Understanding plant density effects on maize growth and development: an important issue to maximize grain yield. Ciência Rural, 31(1), 59-168. doi:10.1590/S0103-84782001000100027

Sarlangue, T., Andrade, F. H., Calviño, P. A., Purcell, L. C. (2007). Why do maize hybrids respond differently to variations in plant density? Agronomy Journal, 99(4), 984-991.
Squire, G. (1990). The physiology of tropical crop production. Wallingford, UK: CABI.

Thomas H. (1992). Canopy survival. In: N. Baker \& H. Thomas (Eds.), Crop Photosynthesis: Spatial and Temporal determinants (pp. 11-41). Amsterdam: Elsevier. doi:10.1016/B978-0-444-89608-7.500095

Tollenaar, M., Daynard, T. B. (1982). Effect of sourcesink ratio on dry matter accumulation and leaf senesence of maize. Canadian Journal of Plant Science, 62(4), 855-860. doi:10.4141/cjps82-128

Uhart, S. A., Andrade, F. H. (1995). Nitrogen and carbon accumulation and remobilization during grain filling in maize under different source/sink ratios. Crop Science, 35(1), 183-190. doi:10.2135/cropsci1995.0011183X003500010034 $\mathrm{x}$

Van Sanford, D., Mackown, C. (1987). Cultivar differences in nitrogen remobilisation during grain filling in soft red winter wheat. Crop Science, 27, 295-300. doi:10.2135/cropsci1987.0011183X002700020035 $\mathrm{x}$

Varlet-Grancher, C., Gautier, H. (1995). Plant morphogenetic responses to light quality and consequences for intercropping (Vol. 93, pp. 231256). Presented at the Symposium International sur 1' Ecophysiologie des cultures Associees Tropicales, Gosier, Guadeloupe, 1-6.

Yin, Y., Wang, Z., He, M., Fu, J., Lu, S. (1998). Postanthesis allocation of photosynthates and grain growth in wheat cultivars as affected by source/sink change. Biologia Plantarum, 41(2), 203-209. doi:10.1023/A:1001854311288

Zhenlin, W., Yanping, Y., Mingrong, H., Hongming, C. (1998). Source-sink manipulation effects on postanthesis photosynthesis and grain setting on spike in winter wheat. Photosynthetica, 35(3), 453459. doi:10.1023/A:1006976605148 